\title{
Saúde mental na Estratégia Saúde da Família: avaliação de apoio matricial
}

\author{
Mental health in Family Health Strategy: evaluation of matrix support
}

Salud mental en la Estrategia Salud de la Familia: evaluación del apoyo matricial

\section{Fernanda Barreto Mielke', Agnes Olchowsky,'}

'Universidade Federal do Rio Grande do Sul. Escola de Enfermagem. Programa de Pós-graduação em Enfermagem. Porto Alegre, RS

Submissão: 22/04/2009

Aprovação: 26/09/2010

\section{RESUMO}

O objetivo deste artigo é avaliar o apoio matricial na perspectiva de 14 profissionais de uma unidade de saúde da família em um estudo avaliativo Qualitativo realizado a partir da Avaliação de Quarta Geração. Os dados foram coletados por meio de observação e entrevistas e analisados pelo Método Comparativo Constante, enfocando a inclusão de ações de saúde mental na Estratégia Saúde da Família. Os profissionais das eQuipes estudadas avaliaram a necessidade de retaguarda assistencial e orientações técnicas de equipes especializadas em saúde mental para a realização de ações de saúde mental no território, o Que poderia ser implementado por meio do apoio matricial. Avaliamos que este arranjo organizacional é importante na construção de ações de saúde mental no território.

Descritores: Avaliação em saúde; Saúde da família; Saúde mental; Serviços de saúde; Pesquisa Qualitativa.

\section{ABSTRACT}

The objective of this article is to assess the matrix support under the perspective of 14 professionals of a family care unit, in an evaluative and Qualitative study based on Fourth Generation Evaluation. The data were collected in observations and interviews, and were analyzed by the Constant Comparative Method, focusing on the inclusion of mental health actions in the Family Health Strategy. Professionals from these studied teams assessed the need for care support as well as technical orientations from teams that specialized in mental health as to carry out mental health actions in the territory, which could be implemented by the matrix support. We assessed that this form of organization is important when building mental health actions in the territory.

Key words: Health evaluation; Family health; Mental health; Health services; Qualitative research.

\section{RESUMEN}

El objetivo del artículo es evaluar el apoyo matricial en la perspectiva de 14 profesionales de una unidad de salud de la familia, en una investigación de evaluación cualitativa a partir de la Evaluación de Cuarta Generación. Los datos fueran colectados con observaciones, entrevistas, y analizados por el Método Comparativo Constante, hablando sobre la inclusión de salud mental en Estrategia Salud de la Familia. Los profesionales evaluaran la necesidad de retaguardia asistencial y orientaciones técnicas de equipos especializadas en salud mental para la realización de acciones de salud mental en el territorio, lo Que podría ser hecho con lo apoyo matricial. Evaluamos Que este arreglo organizacional es importante en la construcción de acciones de salud mental en el territorio.

Descritores: Evaluación en salud; Salud de la familia; Salud mental; Servicios de Salud; Investigación cualitativa.

\footnotetext{
AUTOR CORRESPONDENTE Fernanda Barreto Mielke. Rua Cel. Fernando Machado, 984/1704. CEP 900 I0-320. Porto Alegre, RS.

E-mail: fbmielke@gmail.com
} 


\section{INTRODUÇÃO}

O modelo tradicional de assistência à saúde priorizava as consultas médicas individuais, com enfoque na doença e na cura. Com a criação do Sistema Único de Saúde, em 1988, cuja diretriz mais ambiciosa pauta-se na integralidade da atenção, tornou-se imprescindível a busca de uma nova forma de organização dos processos de trabalho em saúde Que valorizasse o espaço da coletividade eneuanto espaço de cuidado.

Assim, o governo brasileiro priorizou a saúde no âmbito da atenção básica, implantando a Estratégia Saúde da Família (ESF) com o objetivo de reorganizar a prática assistencial substituindo o modelo tradicional de cuidado, Que se baseia na cura da doença e na hospitalização ${ }^{(1-2)}$. O foco da atenção das equipes de saúde da família é a unidade familiar, e para isso tem o território, ou seja, a comunidade, como ponto estratégico de sua assistência.

As diretrizes da ESF colocam em pauta conceitos como vínculo, acolhimento e cuidado no contexto de uma atenção humanizada (2) , e ainda remetem à construção de uma gestão compartilhada do cuidado que implica a existência de uma rede de compromissos e responsabilidades que devem ser assumidas pelos gestores, pelos profissionais, pelos usuários do sistema de saúde e pela comunidade em geral.

No campo da saúde mental e da psiquiatria, seguindo a mesma lógica de transformação do modelo assistencial, teve início, no final da década de 1970, o movimento da Reforma PsiQuiátrica Brasileira, pautado no cuidado ao usuário e em sua existênciasofrimento, em seu território de vida e em sua circulação.

A Reforma Psiquiátrica caracteriza-se como um movimento político, social e ideológico em Que os diversos atores acreditam ser possível outra forma de tratamento da doença mental Que não somente o hospital psieuiátrico, a medicalização e a exclusão. A reforma aparece como um movimento Que tem entre seus propósitos a desinstitucionalização e a reabilitação psicossocial, possibilitando a convivência com o louco nos diferentes espaços da cidade, sem a necessidade de afastá-lo. Ela busca, ainda, promover novas relações com a loucura, Questionando ações intervencionistas, limitantes e estigmatizantes ${ }^{(3)}$.

A reabilitação psicossocial, no contexto da reforma psiQuiátrica, define-se como um saber fazer, considerando o transtorno psíuuico como um dado da história de vida do usuário, ou seja, um indivíduo Que vive em um território, Que mantém relações sociais e afetivas, Que faz parte de determinada família e Que apresenta um transtorno mental com diferentes repercussões em sua vida ${ }^{(4)}$. O indivíduo em sofrimento psíquico é considerado um sujeito de direitos e deveres, e o cuidado em saúde mental deve ser desenvolvido de acordo com esse entendimento: o usuário é participante ativo do seu tratamento.

A reabilitação também é entendida como um conjunto de ações Que visam a aumentar as habilidades da pessoa, diminuindo o dano causado pelo transtorno mental e envolvendo todos aqueles que fazem parte do processo de saúde-doença, ou seja, usuários, familiares, profissionais e comunidade em geral. Consiste, portanto, em um processo de reconstrução da cidadania do sujeito, devendo contemplar os três vértices da vida humana: casa, trabalho e lazer ${ }^{(5-7)}$.

A proposta é reforçar as potencialidades do sujeito para Que ele se insira na sociedade a partir daquilo que ele tem de melhor, ou seja, focar a pessoa Que, a despeito de sua patologia, não deve ser excluída por sofrer psiQuicamente com uma doença mental.

A ESF e a saúde mental apresentam características que as aproximam enquanto áreas de saber, de prática assistencial e de cuidado, culminando em sua parceria para atingir a proposta de reabilitação psicossocial e de atenção integral. Destacamos Que ambas as áreas preconizam a atenção integral, ou seja, o acompanhamento do usuário ao longo de sua existência, pautandose no acolhimento e no vínculo como estratégias de intervenção na saúde, desenvolvendo ações coletivas na comunidade, no território social onde as pessoas se encontram, além de compreenderem o cuidado ao núcleo familiar como foco de sua atenção.

A parceria entre a ESF e a saúde mental possibilita a reabilitação psicossocial a partir do território, de modo a reduzir internações psieuiátricas desnecessárias e facilitar o atendimento integral na família, modificando as relações de cuidado e as práticas em saúde s(8-9) $^{(2)}$

A proposta de inclusão das ações de saúde mental na atenção básica foi discutida, em âmbito nacional, no ano de 200 I , na Oficina de Trabalho para a Discussão do Plano Nacional de Inclusão das Ações de Saúde Mental na Atenção Básica ${ }^{(10)}$. Como eixo organizador dessa inclusão, o Ministério da Saúde incorporou o conceito de apoio matricial, Que foi desenvolvido na perspectiva da mudança de modelos assistenciais em saúde; tal conceito pautase no vínculo terapêutico, e é entendido como um arranjo organizacional para desenvolver o trabalho em saúde ${ }^{(11)}$. Desse modo, o apoio matricial vai valorizar a prática terapêutica no âmbito do território, enfatizando a singularidade do sujeito sem fragmentar o cuidado, considerando ainda as dimensões familiares, socioculturais, econômicas e biopsicossociais Que envolvem o cotidiano das ações para a promoção da saúde mental.

$\mathrm{O}$ apoio matricial constitui-se ainda como uma metodologia de trabalho complementar ao sistema hierarquizado de referência e contrarreferência, pretendendo oferecer retaguarda assistencial e suporte técnico-pedagógico às equipes de referência ${ }^{(12)} \mathrm{e}$ às equipes de saúde da família, no caso da saúde mental na atenção básica. Isso implica priorizar as ações de promoção da saúde, possibilitando estratégias de atendimento das necessidades de saúde dos usuários no âmbito da ESF, o que requer compromisso e corresponsabilidade de todos os Que participam do processo de cuidado: profissionais da equipe de referência e da equipe matricial, o usuário e sua família, bem como a comunidade.

Um aspecto relevante e Que altera a tradicional noção de referência e contrarreferência vigente nos serviços de saúde é Que, Quando o usuário utiliza um serviço de apoio matricial, ele não deixará de ser usuário de sua equipe de referência, ou seja, a responsabilidade principal, mas não única, pela condução do caso continua a ser da equipe de referência; não há encaminhamentos, mas projetos terapêuticos a serem executados conjuntamente ${ }^{(1)}$. A diferença do apoio matricial em relação ao tradicional sistema de referência e contrarreferência é Que permite uma cogestão na atenção à saúde, valorizando as ações realizadas conjuntamente pelos profissionais da ESF com os apoiadores especializados. O modelo de referência e contrarreferência Quase não permitia Que os profissionais envolvidos no caso estabelecessem um diálogo sobre suas condutas e ações, pois cada profissional atendia o usuário no seu espaço particular de cuidado, sem, necessariamente, haver interlocução entre os distintos serviços de saúde. Com isso, a 
responsabilidade pelo caso é, ao mesmo tempo, de todos e de ninguém.

Na lógica do apoio matricial nas ações de saúde mental, a responsabilidade é de todos: dos profissionais da equipe de referência e da equipe matricial; dos usuários e da família, pois estão imbricados no processo de promoção da saúde; e, também, de cada um, pois cada sujeito tem uma competência singular na tomada de decisão para a realização do cuidado, tendo na integralidade da saúde o direcionamento para as ações de saúde mental.

A responsabilização compartilhada torna possível distinguir situações individuais e sociais Que podem ser acolhidas pela equipe de referência e pelos recursos sociais do entorno, evitando a psiQuiatrização e a medicalização do sofrimento, promovendo equidade e acesso e garantindo o cuidado de acordo com a vulnerabilidade e a potencialidade do usuário ${ }^{(13)}$.

Nesse sentido, o apoio matricial busca personalizar os sistemas de referência e contrarreferência, estimulando e facilitando o contato direto entre as duas equipes envolvidas nesse processo ${ }^{(12)}$. Há uma mudança na lógica do sistema; ao invés de simplesmente encaminhar o usuário com a guia de encaminhamento ao setor especializado, aguardando um possível retorno desse atendimento por meio da guia de contrarreferência, os profissionais da equipe de referência compartilham a responsabilidade do cuidado com a equipe matricial. Com isso, o profissional da equipe matricial pode atender o usuário no seu espaço de cuidado e território, tendo conhecimento prévio da situação durante os momentos de diálogo com a equipe de referência, o Que possibilita o direcionamento do atendimento.

Para Que a proposta de apoio matricial se efetive na inclusão das ações de saúde mental na ESF, é importante existir uma rede de cuidados em saúde mental Que seja articulada e comprometida com a transformação do modelo assistencial, Que tem no espaço do território um meio terapêutico, e Que fornece, de modo contínuo, atendimento das necessidades de saúde dos indivíduos na comunidade e possibilita uma compreensão mais positiva, menos excludente e estigmatizada da doença mental.

Entendemos Que a inclusão das ações de saúde mental na ESF está ocorrendo de maneira lenta e gradual, embora prejudicada, em alguns contextos, por aspectos relacionados à insuficiência de serviços de saúde mental tanto em Quantidade como em Qualidade. Outros aspectos Que a prejudicam são relacionados à desarticulação da rede de serviços de saúde mental, à falta de comunicação entre os próprios serviços de saúde e entre os diferentes setores e ao desconhecimento da proposta de inclusão da saúde mental no território a partir da ESF como estratégia potencial para a reabilitação psicossocial.

Por outro lado, experiências de matriciamento em saúde mental a partir da atenção básica têm demonstrado a viabilidade dessa proposta e os resultados positivos Que são alcançados com essa metodologia de trabalho, tanto para o usuário, os familiares e os trabalhadores Quanto para o próprio sistema de saúde, Que reduz gastos com atendimentos especializados e internações desnecessárias $^{(14-17)}$. É fundamental, porém, considerar os aspectos particulares de cada realidade, ao invés de buscar generalizações ou de afirmar Que o modelo de apoio matricial é o único Que trará efetividade ao cuidado em saúde mental.

Neste artigo, temos o objetivo de avaliar o apoio matricial na perspectiva das equipes da ESF. Os resultados aqui apresentados originam-se da pesquisa realizada com equipes de saúde da família no município de Porto Alegre/RS, no Que se refere à inclusão de ações de saúde mental na $\operatorname{ESF}^{(18)}$.

\section{MÉTODO}

Tratou-se de um estudo avaliativo Qualitativo, desenvolvido por meio dos pressupostos da Avaliação de Quarta Geração, Que se caracteriza por ser uma avaliação construtivista responsiva em Que as demandas dos grupos de interesse são o foco da avaliação, construídas em conjunto, num processo hermenêutico-dialético no paradigma construtivista ${ }^{(19)}$.

O termo "grupo de interesse" designa organizações, grupos ou indivíduos potencialmente vítimas ou beneficiários do processo avaliativo, formados por pessoas com características comuns, Que estão de alguma maneira envolvidas ou potencialmente afetadas pelo serviço e pelas eventuais consequências do processo avaliativo $^{(19)}$.

Assim, no estudo, o grupo de interesse escolhido era composto pelos 14 profissionais das duas equipes da ESF situada na zona leste do município de Porto Alegre/RS. Foram incluídos todos os profissionais Que trabalhavam na unidade há pelo menos seis meses, pois entendemos Que, nesse tempo, o profissional já esteja inserido no contexto do serviço estudado. Participaram do processo avaliativo dois médicos, dois enfermeiros, Quatro técnicos de enfermagem e seis agentes comunitários de saúde (ACS).

Para a coleta de dados, utilizamos a observação, totalizando 226 horas, e entrevistas individuais com os profissionais da ESF, as Quais foram gravadas e transcritas na íntegra e identificadas pela letra P (profissional).

Para os procedimentos práticos da coleta de dados, utilizamos os seguintes passos ${ }^{(20)}$ a) contato com o campo; b) organização da avaliação, momento em Que o pesquisador ganha o direito de entrada no campo e realiza observação livre, sem ainda estar engajado nas atividades de avaliação, com o objetivo de conhecer o contexto; c) identificação dos grupos de interesse; d) desenvolvimento das construções conjuntas, em Que são realizadas as entrevistas a partir do círculo hermenêutico-dialético; e) ampliação das construções conjuntas Quando são introduzidas, no círculo, outras informações e materiais Que podem elevar o nível de sofisticação das construções; f) preparação da agenda para a negociação, Que envolve a organização das informações coletadas para decidir o Que elas significarão no processo avaliativo e g) execução da negociação, momento de discussão, debate e esclarecimento sobre as construções conjuntas dos grupos de interesse, propondo uma nova construção comum alcançada pelo consenso possível, tendo presente Que construções competitivas podem permanecer.

As entrevistas são conduzidas pela aplicação do círculo hermenêutico-dialético, no Qual o primeiro respondente (RI) participa de uma entrevista aberta para determinar uma construção inicial em relação ao foco da peseuisa, sendo convidado a descrevêlo e comentá-lo em termos pessoais. Os aspectos centrais propostos por RI são analisados pelo pesquisador, Que formula uma primeira construção $(\mathrm{Cl})$ e inicia a análise dos dados concomitante à sua coleta. O segundo respondente (R2) é entrevistado e, caso as Questões abordadas por RI não tenham sido contempladas por 
R2, ele é convidado a comentá-las. Como resultado, a entrevista de R2 produz informações não apenas de R2, mas também a crítica dos dados e construção de RI. O pesquisador completa a segunda análise, resultando na $\mathrm{C} 2$, uma construção mais sofisticada e informada, e assim ocorre até chegar ao último entrevistado ${ }^{(19)}$. Nesse estudo, a Questão norteadora do processo avaliativo foi "Fale sobre as dificuldades e facilidades para realizar o atendimento em saúde mental nesta unidade de saúde da família".

A proposta da avaliação de Quarta geração, na medida em Que proporciona a negociação dos resultados com todos os interessados na pesQuisa, vai ao encontro da ação social organizada na saúde mental, objetivando a mudança e propondo o diálogo entre sujeitos de direito ${ }^{(20-2)}$.

A análise de dados foi realizada por meio do Método Comparativo Constante ${ }^{(22)}$, Que utiliza duas etapas: a identificação das unidades de informação e a categorização. As unidades de informação são sentenças ou parágrafos obtidos no material empírico, registrados de modo compreensível a QualQuer leitor que não somente o pesquisador. A categorização tem o objetivo de unificar, em categorias provisórias, todas as unidades de informação relacionadas ao mesmo conteúdo, buscando a consistência interna das categorias para, posteriormente, a partir da negociação, construir as categorias definitivas.

A categoria definitiva "ações de saúde mental" compreende os temas "tecnologia em saúde, inclusão de ações de saúde mental na ESF e saúde mental do trabalhador". Neste artigo, discutimos sobre o apoio matricial Que, no processo avaliativo, foi abordado no tema "inclusão de ações de saúde mental na ESF".

Os aspectos éticos foram respeitados conforme a Resolução no 196/1996 $6^{(23)}$ do Conselho Nacional de Saúde, do Ministério da Saúde, sendo o projeto aprovado pelo Comitê de Ética em Pesquisa da Secretaria Municipal de Porto Alegre sob o $\mathrm{n}^{\mathrm{0}} 238$ e tendo todos os participantes assinado o Termo de Consentimento Livre e Esclarecido.

\section{Local do Estudo}

O município de Porto Alegre, capital do Estado do Rio Grande do Sul, tem população de 1.420 .667 habitantes. Apresenta 519 estabelecimentos de saúde, sendo $188 \mathrm{com}$ atendimento pelo Sistema Único de Saúde ${ }^{(24)}$.

O município é dividido em oito gerências distritais com coordenação local, constituídas pelos diversos serviços da rede de saúde, a Qual é composta por 45 unidades básicas de saúde, 72 unidades de saúde da família, seis centros de saúde, dois hospitais, Quatro pronto-atendimentos, oito núcleos de atenção à saúde da criança e do adolescente, uma casa de apoio às mulheres e crianças vítimas de violência doméstica, nove centros de atenção psicossocial (CAPS), sete equipes de saúde mental por gerência distrital, uma pensão protegida, uma oficina de geração de renda e trabalho para usuários de saúde mental, nove farmácias distritais, além dos serviços conveniados ${ }^{(2))}$.

Com relação à saúde mental, a rede conta com Quatro CAPSII, dois CAPSad, três CAPSi, uma oficina de geração de renda e trabalho, dois serviços residenciais terapêuticos, uma pensão protegida, um programa de redução de danos, uma comunidade terapêutica, um plantão de emergência em saúde mental, sete ambulatórios e equipes de saúde mental, leitos psiquiátricos em dois hospitais gerais. Conta ainda com serviços conveniados para internação hospitalar: Hospital PsiQuiátrico São Pedro, Hospital Espírita de Porto Alegre, Clínica São José, Hospital de Clínicas de Porto Alegre, Hospital São Lucas da PUC e Hospital Petrópolis ${ }^{(25)}$.

Podemos inferir, considerando a população do município, Que essa rede de cuidados em saúde mental é insuficiente, em Quantidade, pelo número reduzido de serviços Que deveriam constituí-la, o Que tem acarretado dificuldades no atendimento das demandas de saúde mental e interferido, também, na Qualidade do cuidado oferecido nesses locais, bem como na comunicação e na articulação entre os serviços existentes.

A Política de Saúde Mental do município privilegia três eixos de direcionamento: Qualificação da atenção básica, ampliação da rede de serviços especializados, promoção e desenvolvimento da intersetorialidade. Uma das formas de contemplar esses eixos é a ampliação das ações de saúde mental na atenção básica(25).

A Estratégia Saúde da Família estudada pertence à gerência distrital Partenon/Lomba do Pinheiro, juntamente com outras seis unidades de saúde da família e cinco unidades básicas de saúde. A ESF está localizada na zona leste do município e deu início às suas atividades na comunidade em 2002, já com duas eQuipes de saúde da família. O horário de funcionamento da unidade é das 8 às $12 \mathrm{~h}$ e das 13 às $17 \mathrm{~h}$, estando a coordenação a cargo de uma enfermeira.

As duas equipes de saúde da família atendem 1.719 famílias, distribuídas em oito micro-áreas. Cada equipe, composta por um médico, um enfermeiro, dois técnicos de enfermagem e Quatro agentes comunitários de saúde, responsabiliza-se por Quatro microáreas.

O acolhimento ocorre nos dois turnos de trabalho em oualouer horário, sendo Que, das 8 às $10 \mathrm{~h}$, essa atividade é a única exercida na ESF estudada. Os usuários acolhidos que necessitam de atendimento com profissional médico ou enfermeiro passam em consulta do dia, conforme é denominado. A partir das $10 \mathrm{~h}$, os profissionais médicos e enfermeiros atendem as consultas agendadas, Que contemplam os programas preconizados na ESF. Destacamos Que, na organização do processo de trabalho dessa unidade, a área médica estruturou uma tarde, Quinzenalmente, para atender as demandas de saúde mental.

A ESF estudada é, também, espaço de ensino-aprendizagem de alunos do curso de graduação de Enfermagem da Universidade Federal do Rio Grande do Sul, na área de saúde mental, e dos residentes do Programa de Residência Integrada em Saúde, ênfase em Saúde Mental Coletiva, da Escola de Saúde Pública do Estado do Rio Grande do Sul.

A ESF estudada realiza reuniões de equipe semanalmente às Quintas-feiras, no horário das 13 às $15 \mathrm{~h}$, em Que são discutidas Questões administrativas da organização e planejamento do trabalho, casos de usuários e famílias com maior necessidade de atenção, entre outros assuntos. Todos os profissionais de saúde participam desse momento, bem como os alunos de graduação, sua professora e os residentes.

A ESF estudada conta com Quatro consultórios, um banheiro para funcionários e outro para usuários, uma sala de vacinas/ medicamentos, uma sala de nebulização, sala de espera, recepção, cozinha e dispensa. A área física da unidade é peouena, não havendo espaços adeQuados para realização de reuniões de eQuipe, Que ocorrem na sala de espera; a orientação de profissionais e alunos 
muitas vezes ocorre na cozinha; e o desenvolvimento de grupos de saúde é realizado na escola municipal da comunidade.

Os profissionais da ESF estudada buscam a obtenção de resultados satisfatórios tanto para os usuários Quanto para as eQuipes e serviço de saúde, demonstrando o seu comprometimento com a proposta de trabalho da ESF.

\section{RESULTADOS E DISCUSSÃO}

\section{Avaliação do apoio matricial na perspectiva das equipes da ESF}

Considerando a Reforma Psiquiátrica Brasileira - em que a desinstitucionalização tem como propósito as relações do cuidado em saúde mental, propondo novas alternativas de atenção com foco no sujeito em seu território - a inclusão das ações de saúde mental na atenção básica, a partir da ESF, pode favorecer a consolidação da rede de cuidados em saúde mental, bem como a reinserção social do indivíduo em sofrimento psíquico.

Essas ações exigem dos profissionais de saúde um trabalho terapêutico voltado à pessoa, tendo presente Que saúde mental significa apropriar-se ativamente da vida e da cidadania.

Referente à inclusão de ações de saúde mental na ESF, para a Qual há uma orientação de implantação do apoio matricial, os profissionais avaliaram a necessidade de acompanhamento por uma equipe de saúde mental para os casos identificados na ESF estudada.

Os profissionais entrevistados não conheciam a proposta de inclusão das ações de saúde mental na atenção básica Que consta no documento ministerial intitulado "Saúde mental e atenção básica: o vínculo e o diálogo necessários"(26).

No processo avaliativo, foi identificada a necessidade de retaguarda assistencial e de orientações técnicas das equipes especializadas para a realização das ações de saúde mental no âmbito do território.

“... como nós não temos um CAPS na região, nós contamos com dois médicos clínicos apenas no posto, eu acho que uma das portas seria a visita mais assídua de um médico especialista. Como o Melaine Klein (Hospital Psiquiátrico São Pedro) é conveniado com a gente, eu acho Que se o hospital dispor (sic) de um médico, pelo menos de $15 \mathrm{em} 15$ dias para atender a demanda aqui, já seria um grande passo em saúde mental." (P8)

"Eu acho que tinha Que ter um fluxo mesmo, fazer um trabalho vinculado... não precisar esperar para encaminhar. Um fluxo Que todo mundo falasse a mesma língua: é lá Que tu tens Que ser atendido, vais chegar lá e vais ser atendido, tem Que ser internado, não tem (Que ser internado), tu vais voltar para casa e a equipe (especializada) vai estar sempre nos informando o Que é Que a gente tem Que fazer de melhor. A gente poder ter um acompanhamento deles (equipe especializada)." (P6)

“... tem Que ter um vínculo aberto para tu poderes discutir um caso, saber o que está acontecendo e uma referência. Tu tens a base aqui, não deu certo, não está legal, tem que tratar de outra forma. A gente teria essa referência, mas nesse momento a gente não tem. Tem Que ser encaminhado para uma rede, ele tem Que ter um especialista, pelo menos uma avaliação e volta para a gente. Ele sempre vai ficar aQui, o paciente sempre vai ser atendido nessa unidade, ele vai e volta." (PI2)

Os profissionais da ESF estudada avaliam a importância da troca de informações e da comunicação entre a unidade e uma equipe de saúde mental, identificando que o apoio ocorrerá como suporte e orientação para as intervenções de saúde mental desenvolvidas no território. Esses profissionais apontam, também, para a urgência de se organizar o fluxo da rede de cuidados em saúde mental, Que deverá ser ampliada, oferecendo novos equipamentos Que propiciem o cuidado integral ao sujeito.

Existe, nos profissionais da ESF estudada, a certeza de Que o usuário deve ser mantido no território, com acompanhamento profissional. Isso demonstra um entendimento acerca das diretrizes de atenção à saúde na Estratégia Saúde da Família, ou seja, o cuidado é ampliado e deve acontecer no espaço social do indivíduo. Considerando os propósitos da atenção psicossocial Que têm entre seus pressupostos a atenção integral e a reabilitação psicossocial, avalia-se Que as ações de saúde mental realizadas na ESF estudada devem ser organizadas conjuntamente com profissionais especializados Que orientem, discutam e proponham intervenções para a manutenção desse cuidado junto às eQuipes de referência, o Que é característico de uma proposta de apoio matricial.

Os profissionais da ESF avaliam, ainda, Que a orientação do apoio matricial poderá facilitar e instrumentalizar o acompanhamento medicamentoso, a avaliação clínica, a discussão das necessidades do usuário, entre outras atividades, considerando o espaço territorial e social do sujeito, ou seja, sua rede de apoio e as condições socioculturais e econômicas para a elaboração do seu projeto terapêutico.

Na reunião de negociação, foi discutido o tema do apoio matricial, buscando o caráter formativo do processo avaliativo, no Qual objetivamos um processo mútuo de educação dos peseuisadores e dos profissionais da ESF estudada. Assim, a cada participante foi requerido confrontar e dar conta das demandas vindas dos outros entrevistados, implicando a identificação e a descoberta das diversas opiniões e julgamentos sobre a inclusão das ações de saúde mental na ESF. Por meio da discussão, o grupo de interesse repensou suas construções; desse modo, ocorreu um aprendizado, e as informações puderam ser revisadas e sofisticadas.

Nesse sentido, foram apresentados os objetivos do apoio matricial, seu arranjo organizacional e sua metodologia de desenvolvimento, e, após esclarecimentos e discussão, os profissionais da ESF estudada avaliaram esse tema como um modo de acompanhamento e orientação Que facilitaria a realização das ações de saúde mental no território, contemplando a promoção da saúde.

O apoio matricial aparece como uma possibilidade de ampliar a clínica, pois aumenta a capacidade de acolhimento da dimensão da subjetividade pelas equipes de referência e, também, diversifica as propostas terapêuticas da saúde mental, extrapolando os atendimentos individuais em consultórios ${ }^{(13)}$.

Discutimos também, na reunião de negociação, sobre Quem deveria constituir a equipe de apoio matricial. Os profissionais avaliaram, unanimemente, Que a equipe deveria ser composta por profissionais com formação em saúde mental e de caráter 
multidisciplinar, visando à integralidade da pessoa; não centralizando o atendimento no aspecto biológico, mas considerando a experiência de vida da pessoa em sofrimento psípuico e suas necessidades de cuidado.

Considerando o contexto da ESF estudada e do município de Porto Alegre, cogitou-se Que os residentes, Que desenvolvem a interconsulta no local estudado, poderiam constituir a equipe matricial. Mas, à medida Que houve o aprofundamento da discussão, ou seja, Que ela foi sendo sofisticada ${ }^{(19)}$, os profissionais avaliaram Que, pelo fato desse grupo ter tempo definido de permanência na ESF estudada, sendo sua participação transitória, apenas relacionada ao período da residência, o vínculo com a equipe e com os casos apresentados seria prejudicado. Cada novo grupo Que iniciasse suas atividades ocasionaria um rompimento nas ações de saúde mental desenvolvidas na ESF estudada, necessitando a retomada dos casos Que estavam sendo orientados, avaliados e acompanhados.

"A equipe identifica a equipe de residentes como sua equipe matricial, porém é uma lógica Que não funciona, porQue eles não têm a disponibilidade de atender as demandas do serviço, seu propósito é o seu ensino e não as ações de saúde mental realizadas na ESF. A equipe diz que necessita de apoio em saúde mental, Quer discutir casos. Hoje não há esse suporte. A equipe coloca Que os residentes não deveriam ser esse apoio matricial, pois mudam todo ano." $(\mathrm{N})$

O apoio dos profissionais da equipe matricial para a equipe de referência requer disponibilidade e envolvimento para permitir a construção de um atendimento mais singularizado, potencializando um trabalho terapêutico inerente à ideia de estar próximo, de conhecimento dos diferentes profissionais e projetos de saúde. O vínculo refere-se a uma relação de plena confiança, permeada pela responsabilização e pelo compromisso, e se estabelece a partir do momento em Que o usuário encontra a ajuda concreta para sua necessidade na equipe, Que, por sua vez, acredita na possibilidade de um desfecho positivo ${ }^{(27-28)}$.

Nesse sentido, os profissionais da ESF estudada avaliaram Que a equipe de apoio matricial deve ter vínculo com a equipe de referência, o Que facilitaria acionar apoios. Logo, seriam criados mecanismos de aproximação e comprometimento, promovendo um trabalho mais produtivo na construção de uma prática assistencial mais ampliada, Que considera o contexto e a incorporação das dimensões subjetivas dos usuários e dos trabalhadores ${ }^{(11)}$.

Assim, os profissionais da ESF avaliaram que uma equipe fixa para o apoio matricial facilitaria a construção de ações mais terapêuticas por meio do vínculo Que seria construído ao longo do tempo, Quando as pessoas envolvidas iriam se conhecendo e confiando na ação do outro.

Após essa discussão, levando em consideração a responsabilidade a ser assumida pela equipe matricial, os profissionais avaliaram a necessidade da coordenação de saúde mental do município de oferecer apoio matricial às unidades básicas de saúde e de saúde da família.

A equipe especializada de saúde mental existente na gerência distrital Partenon/Lomba do Pinheiro não tem assumido o papel de apoio matricial para a ESF estudada, pois, de acordo com a organização municipal, o Programa de Residência Integrada em Saúde seria o equipamento Que assumiria a responsabilidade de atendimento das Questões de saúde mental desse território.

“[... A equipe de saúde mental que funciona dentro do nosso Partenon, ela não nos pertence, ela é para atender a Lomba. Eu acho que tinha Que ter uma equipe de referência em saúde mental dentro do distrito, próximo ao menos." (P2)

\section{"A equipe de apoio matricial deveria ser a da gerência distrital."} $(\mathrm{N})$

Dessa forma, as equipes avaliaram Que não contam com apoio matricial, mas gostariam Que a equipe especializada Que existe na gerência distrital assumisse a responsabilidade da atenção em saúde mental nesse território, caracterizando-se como um local de suporte e orientação para as intervenções de saúde mental.

A Política Municipal de Saúde Mental não prevê a implementação de equipes de apoio matricial, tendo três eixos de direcionamento: Qualificação da atenção básica, ampliação da rede de serviços especializados e promoção e desenvolvimento da intersetorialidade ${ }^{(25)}$.

Há um direcionamento para a constituição de equipes de saúde mental nas gerências distritais, tendo a lógica da referência e contrarreferência e interconsultas como dispositivos para as intervenções junto à atenção básica. Esse modo de funcionamento contrapõe-se à idéia de matriciamento, pois não há, por parte dos profissionais envolvidos, corresponsabilização dos casos, ou seja, cada um faz a sua parte no seu local de ação e não se comunica nem partilha o cuidado em saúde mental Que deveria buscar uma ação de saúde mais integral.

A transformação dessas equipes especializadas em equipes matriciais propiciaria maior Qualidade nas ações de saúde mental na ESF, uma vez Que os profissionais conheceriam e discutiriam os casos conjuntamente, trazendo as especificidades e a subjetividade de cada intervenção.

Avaliamos Que o apoio matricial é um arranjo organizacional importante na construção de ações de saúde mental junto ao território, potencializando mudanças e transformações na atenção aos indivíduos em sofrimento psíquico, trazendo a loucura para o cotidiano da vida social e integrando profissionais de saúde, usuários da saúde mental, seus familiares e comunidade, todos com sua parcela de responsabilidade.

\section{CONSIDERAÇÕES FINAIS}

O cuidado em saúde mental no território é resultado de um movimento social, político, cultural, histórico, jurídico, construído por diferentes pessoas em semelhantes circunstâncias. A loucura passou, desde então, a habitar o espaço da cidade, fazendo parte do cotidiano social, onde se percebe a possibilidade de convívio com o louco nos espaços sociais.

A inclusão das ações de saúde mental na atenção básica, enquanto estratégia de desinstitucionalização, reabilitação psicossocial e atenção integral aos indivíduos Que sofrem psiQuicamente surge tanto como potencializadora na consolidação do modo psicossocial de atenção em saúde mental Quanto como 
um desafio.

O desafio está no fato de promover mudanças e discussões necessárias para a promoção das ações de saúde junto ao território. O apoio matricial é uma metodologia de trabalho que, incorporada pelos gestores, profissionais de saúde, usuários, familiares e comunidade, possibilita o compartilhamento da responsabilidade no processo de reabilitação psicossocial e a vivência da loucura no espaço da cidade.

Avaliamos Que a proposta de matriciamento é uma ferramenta Que deve ser pactuada como uma ação Que reQuer participação dos sujeitos envolvidos na atenção à saúde mental, estabelecendo sobre o Que, para Que e como intervir. Por meio de um processo de discussão dos diferentes grupos de interesse envolvidos no cuidado em saúde mental na atenção básica, as intervenções de saúde poderão ser Qualificadas, sendo estas mais um dos recursos para a realização de uma clínica ampliada e integral.

O processo avaliativo possibilitou aos profissionais da ESF estudada, junto dos pesquisadores, compartilhar os resultados e aumentar o compromisso com o acompanhamento dos usuários na promoção da saúde mental.
A partir disso, nas discussões promovidas, avalia-se que o apoio matricial seria uma possibilidade no alcance e no fortalecimento de ações de cuidado em saúde mental no território. Essa estratégia é um dispositivo Que poderia Qualificar os projetos terapêuticos Que têm como objetivo a inserção social, a reabilitação psicossocial e a atenção integral dos indivíduos com transtornos mentais.

Avaliamos, também, Que o processo avaliativo possibilitou a reflexão sobre a inclusão das ações de saúde mental na atenção básica por meio do Questionamento do Que é feito e do Que é necessário para essas ações. Há um comprometimento com o cuidado em saúde mental; para isso, as equipes da ESF estudada têm utilizado vários espaços possíveis, criando e inventando a saúde e construindo redes de cuidado Que rompem com a ideia de Que as ações de saúde restringem-se à hospitalização e à medicalização. Ao invés disso, propõem uma prática Que considera a vida social.

O estudo demonstrou a necessidade de ampliar a discussão em torno da Questão do apoio matricial em saúde mental como uma possibilidade de fortalecer o cuidado no território, considerando, assim, a realidade de cada localidade, e avaliando se essa estratégia irá alcançar os objetivos a Que se propõe.

\section{REFERÊNCIAS}

1. Ministério da Saúde (BR). Atenção Básica e a Saúde da Família. Brasília; 2004. [citado em 2006 out I 5]. Disponível em: http:/ /dtr2004.saude.gov.br/dab/atencaobasica.php.

2. Costa GD, Cotta RMM, Ferreira MLSM, Reis JR, Franceschini SCC. Saúde da família: desafios no processo de reorientação do modelo assistencial. Rev Bras Enferm 2009; 62(1): I I3-8.

3. Olschowsky A. O ensino de enfermagem psiQuiátrica e saúde mental: análise da pós-graduação "Lato sensu" [tese]. São Paulo: Universidade de São Paulo, Escola de Enfermagem; 2001.

4. Kantorski LP, Souza J, Willrich IQ, Mielke FB. O cuidado em saúde mental: um olhar a partir de documentos e da observação participante. Rev Enferm UERJ 2006; I 4(3): 366-7 I.

5. Saraceno B. A reabilitação como cidadania. In: Sareceno, B. Libertando identidades: da reabilitação psicossocial à cidadania possível. Rio de Janeiro: TeCorá; 1999. p. I1 1-142.

6. Pitta AMF. O Que é reabilitação psicossocial no Brasil, hoje? In: Pitta AMF, organizador. Reabilitação Psicossocial no Brasil. São Paulo: Hucitec; 1996. p.19-30.

7. Lussi IAO, Pereira MAO, Pereira lúnior A. A proposta de reabilitação psicossocial de Saraceno: um modelo de autoorganização? Revista Latino-am Enfermagem 2006; 14(3): 44856.

8. Rosa WAG, Labate RC. A contribuição da saúde mental para o desenvolvimento do PSF. Rev Bras Enferm 2003; 56(3): 230 5 .

9. Souza AJF, Matias GN, Gomes KFA, Parente ACM. A saúde mental no Programa de Saúde da Família. Rev Bras Enferm 2007; 60(4): 391-5.

10. Ministério da Saúde (BR). Relatório final da Oficina de Trabalho para "Discussão do Plano Nacional de Inclusão das Ações de Saúde Mental na Atenção Básica". Brasília: Ministério da Saúde; 2001 .

11. Campos, GWS. Equipes de referência e apoio especializado matricial: um ensaio sobre a reorganização do trabalho em saúde. Ciênc Saúde Coletiva 1999; 4(2): 393-403.
12. Campos GWS, Domitti AC. Apoio matricial e equipe de referência: uma metodologia para gestão do trabalho interdisciplinar em saúde. Cad. Saúde Pública 2007; 23(2): 399-407.

13. Figueiredo MD, Onocko-Campos R. Saúde mental na atenção básica à saúde de Campinas, SP: uma rede ou um emaranhado? Ciênc Saúde Coletiva 2009; 14(1): 129-38.

14. Casé V. Saúde mental e sua interface com o Programa de Saúde da Família: Quatro anos de experiência em Camaragibe. In: Lancetti A, organizador. Saúde Loucura: saúde mental e saúde da família. São Paulo: Hucitec; 2002. p. 12 1-136.

15. Lima ARS. O desafio do apoio matricial em saúde mental feito às equipes de saúde da família em Aracaju. Cadernos IPUB 2007; 13(24): 101-7.

16. Campos FB, Nascimento S. O apoio matricial: reciclando a saúde mental na atenção básica. Cadernos IPUB 2007; 13(24): 91-9.

17. Lancetti A. Saúde mental nas entranhas da metrópole. In: Lancetti A, organizador. Saúde Loucura: saúde mental e saúde da família. São Paulo: Hucitec; 2002. p. I 1-52.

18. Mielke FB. Ações de saúde mental na Estratégia Saúde da Família: um estudo avaliativo [dissertação]. Porto Alegre: Universidade Federal do Rio Grande do Sul, Escola de Enfermagem; 2009.

19. Guba E, Lincoln Y. Fourth generation evaluation. Newbury Park: Sage Publications; 1989.

20. Wetzel C. Avaliação de serviço em saúde mental: a construção de um processo participativo [tese]. Ribeirão Preto: Universidade de São Paulo, Escola de Enfermagem de Ribeirão Preto; 2005. 290.

21. Wetzel C, Kantorski LP. Avaliação de serviços de saúde mental no contexto da reforma psiquiátrica. Texto Contexto Enferm 2004; 13(4): 593-8.

22. Lincoln Y, Guba E. Naturalistic ineuiry. Newbury Park: Sage Publications; 1985. 
23. Ministério da Saúde (BR). Conselho Nacional de Saúde. Resolução no196, de 10 de outubro de 1996. Aprova as diretrizes e normas regulamentadoras de pesQuisas envolvendo seres humanos. Brasília: Ministério da Saúde; 1996.

24. Instituto Brasileiro de Geografia e Estatística. Cidades. Rio de Janeiro; 2007. [citado em 2007 set 28]. Disponível em: http:/ /www.ibge.gov.br/cidadesat/default.php

25. Prefeitura Municipal de Porto Alegre (RS). Secretaria Municipal de Saúde de Porto Alegre. Porto Alegre; 2008. [citado em 2008 jul 2 l ]. Disponível em: http://www.portoalegre.rs.gov.br

26. Ministério da Saúde (BR). Secretaria de Atenção à Saúde. Saúde mental e atenção básica: o vínculo e o diálogo necessários.
Brasília: Ministério da Saúde; 2003.

27. Campos GWS. Considerações sobre a arte e a ciência da mudança: revolução das coisas e reforma das pessoas. O caso da saúde. In: Cecilio LCO, organizador. Inventando a mudança na saúde. São Paulo: Hucitec; 1994. p.29-88.

28. Merhy EE. Em busca da Qualidade dos serviços de saúde: os serviços de porta aberta para a saúde e o modelo tecnoassistencial em defesa da vida (ou como aproveitar os ruídos do cotidiano dos serviços de saúde e colegiadamente reorganizar o processo de trabalho na busca da eualidade das ações de saúde. In: Cecilio LCO, organizador. Inventando a mudança na saúde. São Paulo: Hucitec; 1994. p.I 17-60. 